\title{
阿托伐他汀的合成
}

\author{
徐 力 $a$ 王少华*, $a$ 李 辉 $b$

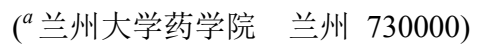 \\ ( 兰州大学化学化工学院 兰州 730000)
}

\begin{abstract}
摘要 阿托伐他汀钻(HMG-CoA 还原酶选择性抑制剂)作为全世界应用最广泛的降胆固醇药物之一, 有关其合成方法 的研究吸引了化学家们的广泛关注. 以廉价易得的 $(R)-2$-氯甲基代环氧乙烷为原料, 经过 13 步反应, 以 $9 \%$ 的总收率成 功地合成了阿托伐他汀, 为相应的仿制药的生产提供了新的路线选择.

关键词 阿托伐他汀钙; 降胆固醇药物; 有机合成
\end{abstract}

\section{Total Synthesis of Atorvastatin}

\author{
$\mathrm{Xu}, \mathrm{Li}^{a} \quad$ Wang, Shaohua ${ }^{*, a} \quad \mathrm{Li}, \mathrm{Hui}^{b}$ \\ ( ${ }^{a}$ School of Pharmacy, Lanzhou University, Lanzhou 730000) \\ ( ${ }^{b}$ School of Chemistry and Chemical Engineering, Lanzhou University, Lanzhou 730000)
}

\begin{abstract}
Atorvastatin calcium (HMG-CoA reductase inhibitor) is the most widely used cholesterol lowering drugs. Its synthesis has attracted broad attention of synthetic chemistry community. In this study, a new strategy has been developed toward the synthesis of atorvastatin in 13 steps and $9 \%$ overall yield.

Keywords atorvastatin calcium; cholesterol lowering drug; organic synthesis
\end{abstract}

心脑血管疾病是一种严重威胁人类健康(特别是中 老年人)的最常见、致死率最高的疾病之一. 据统计, 全 世界每年死于心脑血管疾病的人数已高达 1500 万人 ${ }^{[1]}$, 居各种死因首位. 而在所有的心血管疾病中, 血脂异常, 特别是血清总胆固醇(TC)水平的异常升高则是这类疾 病的一个重要诱因. 考虑到人体内部约 $70 \%$ 的 TC 是内 源性的, 因此, 通过选择性地抑制胆固醇的生物合成来 调节体内血脂含量便成为预防和治疗相关心血管疾病 的一个可行的策略. 相应的, 基于此策略, 很多的他汀 类药物被开发出来. 其中, 阿托伐他汀钙 (Atorvastatin Calcium)又名立普妥(Lipitor), 化学名 $(3 R, 5 R)-7-[2-(4-$ 氟苯基)-3-苯基-4-(苯胺基甲酰基)-5-(2-丙基)吡咯-1基]-3,5-二羟基庚酸钲(图 1), 是一种非常有效的降胆固 醇药物. 作为一种脂溶性物质, 阿托伐他汀钙可以以开 放酸形式进入人体, 在肝脏组织内通过高选择性地抑制 3-羊基-3-甲基-戊二酰辅酶 A 还原酶 (HMG-CoA reductase)的活性，从而抑制体内胆固醇的合成. 它通过

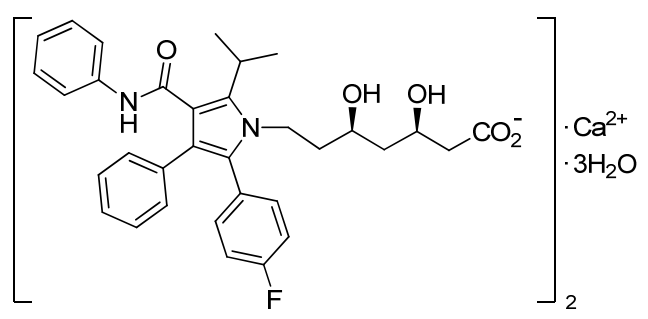

图 1 阿托伐他汀钙的结构

Figure 1 Structure of atorvastatin calcium

有效地促进低密度脂蛋白的摄取及分解代谢，达到降低 血浆中胆固醇脂肪蛋白的水平, 同时升高高密度脂肪蛋 白的水平 ${ }^{[2 \sim 5]}$. 该药物最早由华纳兰伯特 (现并入辉瑞) 研制, 并于 1997 年美国上市销售. 临床研究结果表明, 阿托伐他汀钙是唯一被证明在心血管疾病治疗中优于 血管重建术的调脂药物, 并且对骨质疏松症、痴呆、心 脏病和糖尿病都有一定的疗效. 而且, 同其它他汀类药 物相比, 它的不良反应较少, 因此, 是高血脂患者的首

\footnotetext{
* E-mail: wangshh@1zu.edu.cn

Received February 5, 2015; revised February 14, 2015; published online March 4, 2015.
}

Project supported by the National Natural Science Foundation of China (Nos. 21202073, 21472077) and the Fundamental Research Funds for the Central Universities (Nos. lzujbky-2012-216, lzujbky-2014-k20).

国家自然科学基金(Nos. 21202073, 21472077)和兰州大学中央高校专项基金(Nos. lzujbky-2012-216, 1zujbky-2014-k20)资助项目. 
选用药，药物的销售额也一度居世界畅销药品首位. 由 于该药物的关键专利(US 5273995)已于 2011 年 6 月到 期 ${ }^{[6]}$, 因此, 通过开发新的阿托伐他汀钙合成路线和工 艺技术，来实现相关方制药的生产必将是该药物的下一 个研究重点之一, 同时也具有重要的社会价值.

阿托伐他汀钙的母体结构是阿托伐他汀, 该分子由 一个多取代的吡咯环构成, 其中侧链片段上具有两个二 级羟基的手性中心. 相应的，该分子的合成重点在于多 取代吡咯环的构建及两个立体中心的引入. 虽然, 目前 对于该分子的合成方法较多 ${ }^{[7 \sim 9]}$, 但大多都是基于经典 的 Paal-Knorr 反应模式, 通过侧链 ATS-9 片段(6-氨乙 基-2,2-二甲基-1,3-二氧六环-4-乙酸叔丁酯)与 M4 片段 [4-氟- $\alpha$-(2-甲基- 1 -氧代丙基)- $\gamma$-氧代- $N, \beta$-二苯基苯丁酰 胺]的环化反应来构筑所需的多取代吡咯结构, 进而水 解得到阿托伐他汀 (Eq. 1). 由于 M4 片段的合成方法已 经比较成熟, 因此, 在以上的合成策略中, 大多数针对 阿托伐他汀分子的合成研究都是围绕着 ATS-9 片段的 新的合成策略的研究所开展的. 通过文献调研我们发 现, 在这些合成策略当中, 一级氨基基团的引入大多数 都是通过腈基参与的亲核取代反应及后续的雷尼镍 (Raney-Ni) 催化的氢化反应来实现 ${ }^{[10,11]}$. 考虑到 Raney-Ni 的危险性和对环境的危害, 以及氢气的使用, 这些都会对后续的大规模生产带来一定的安全隐患. 基 于此, 本文发展了一条新的高效、经济的 ATS-9 片段的 合成路线, 并基于此路线最终完成了阿托伐他汀分子的 合成.
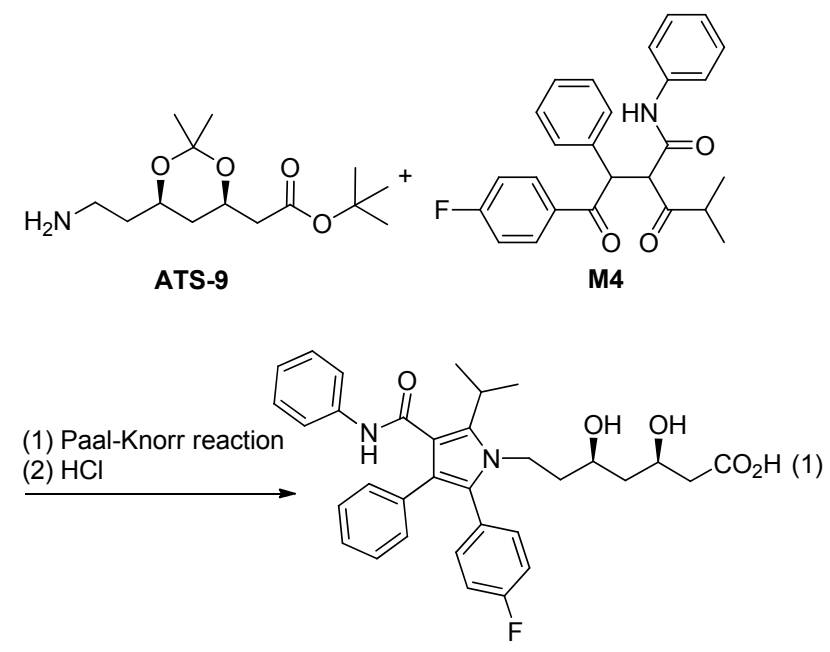

\section{1 结果与讨论}

基于我们最初的想法，期望通过其他的非腈基的方 式引入所需的一级胺结构单元. 经过相应的反合成分 析，叠氮基团成为了我们的首选. 主要基于如下两点考 虑: 首先, 该基团的引入比较方便，通过简单的亲核取 代反应便可实现, 而且, 此类反应的收率通常都比较高;
其次, 由叠氮基团到氨基的化学转化的方法也比较成 熟，除了常见的催化氢化之外，还可以通过 Staudinger 反应来实现 ${ }^{[12]}$, 由于该方法使用廉价易得的三苯基膦作 为还原剂, 不但可以以很高的收率实现叠氮的还原, 同 时可以避免 Raney-Ni 和氢气的使用, 更有利于后续的 规模化生产. 相应的, 对于 ATS-9 片段的反合成分析如 下(Scheme 1): ATS-9 片段可以由化合物 1 经 Staudinger 反应还原分子中的叠氮基团得到; 化合物 1 可又由醛 2 经醛羰基的还原及后续的一级羟基的亲核取代反应获 得; 醛 2 则可以从酮酸酯 3 出发, 经酮羰基的立体选择 性还原，1,3-二羟基的官能团保护，双键的臭氧化断裂 来获得; 而酤酸酯 3 可以通过酯 4 与乙酰叔丁酯的 Dieckmann 缩合反应来获得; 最后酯 $\mathbf{4}$ 可以从廉价易得 的工业原料 $(R)-2$-氯甲基代环氧乙烷(5)经过一些常规的 化学转化获得. 同时, 需要指出的是, 通过该合成策略 ATS-9 片段具有两个立体中心可以从化合物 5 中的立体 中心经不对称诱导得到, 因此, 整个合成过程中避免了 不对称催化反应的使用.
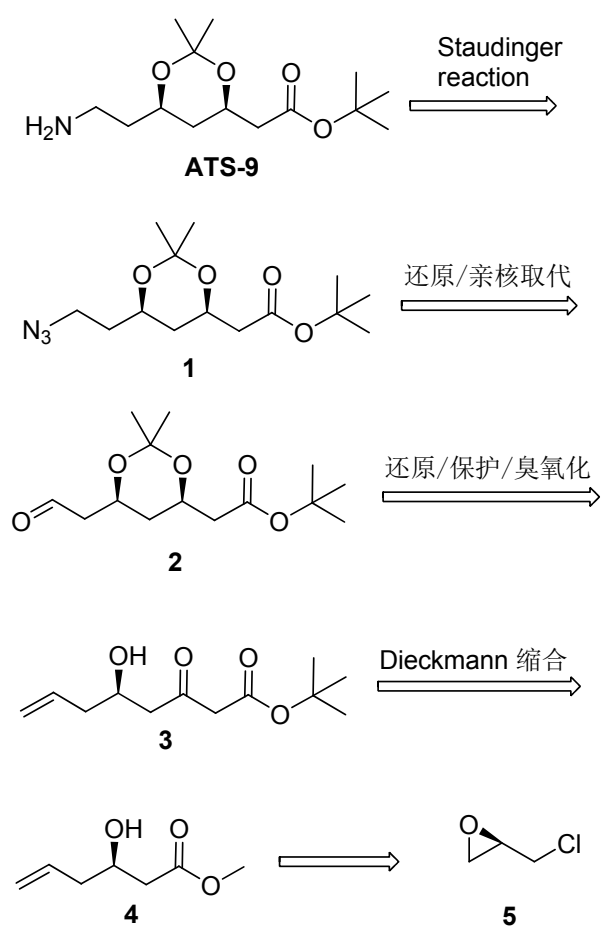

图式 1 逆合成分析

Scheme 1 Retrosynthetic analysis

结合上述的逆合成分析, 如 Scheme 2 所示, 以廉价 易得的 $(R)-2$-氯甲基代环氧乙烷(5)为起始原料, 在氯化 亚铜的催化下，与乙烯基溴化镁经环氧亲核加成开环得 到高烯丙醇 $6{ }^{[13]}$ 高烯丙醇 6 与氰化钠在水相中成功地 通过腈基的亲核取代反应以 $93 \%$ 的收率获得二级醇 7 . 化合物 7 在 TMSCl 作用下在甲醇中发生氰根的水解得 
到甲酯 $\mathbf{4}^{[14]}$. 接下来, 化合物 4 成功地与乙酸叔丁酯在 二异丙基氨基锂作用下经 Dieckmann 缩合反应得到酮 酸酯 $\mathbf{3}^{[15]}$. 然后，化合物 $\mathbf{3}$ 在二乙基甲氧基硼烷的作用 下与硼氢化钠反应 ${ }^{[16]}$, 成功地实现了酮羰基的立体选 择性还原, 得到 1,3-二差基化合物 8 的同时, 构建了阿 托伐他汀所需的两个立体中心. 化合物 $\mathbf{8}$ 经缩丙酮保护 羟基、臭氧化切断碳碳双键便得到了预期的产物醛 2 . 按照最初的计划, 醛 2 经过四步常规反应, 即硼氢化钠 还原羰基、 $\mathrm{OMs}$ 保护一级羟基、 $\mathrm{NaN}_{3}$ 的亲核取代反应 及 Staudinger 反应还原 $\mathrm{N}_{3}$ 基团顺利地得到 ATS-9 ${ }^{[17]}$. 在 得到 ATS-9 片段之后, 将其与 M4 片段进行 Paal-Knorr 环化反应构筑多取代吡咯环结构, 然后在盐酸作用下发 生叔丁酯的水解及缩丙酮保护基的脱除反应，顺利地以 $9 \%$ 总收率得到了预期产物阿托伐他汀 ${ }^{[17]}$.

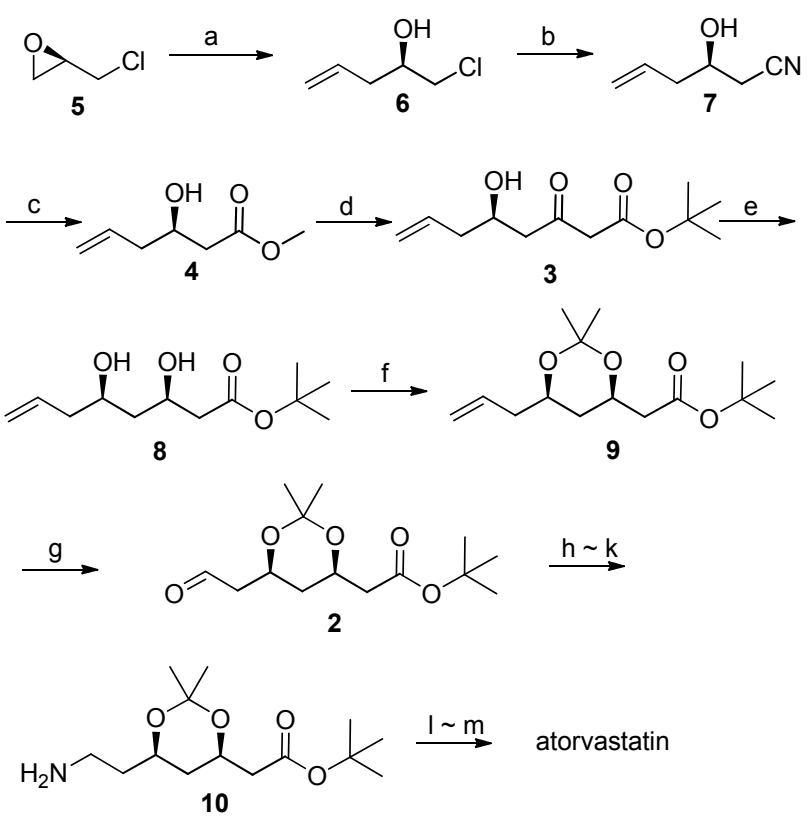

Reagents and conditions: (a) $\mathrm{CH}_{2} \mathrm{CHMgBr}, \mathrm{CuCl}, \mathrm{THF},-20{ }^{\circ} \mathrm{C}, 2$ h, 91\%; (b) $\mathrm{NaCN}, \mathrm{H}_{2} \mathrm{O}, 30{ }^{\circ} \mathrm{C}, 12 \mathrm{~h}, 93 \%$; (c) $\mathrm{TMSCl}, \mathrm{CH}_{3} \mathrm{OH}$, reflux, $15 \mathrm{~h}, 76 \%$; (d) LDA, $\mathrm{CH}_{3} \mathrm{CO}_{2} \mathrm{Bu}-t$, THF, $-78{ }^{\circ} \mathrm{C}, 45 \mathrm{~min}, 65 \%$; (e) $\mathrm{Et}_{2} \mathrm{BOMe}, \mathrm{NaBH}_{4}, \mathrm{THF}, \mathrm{MeOH},-78{ }^{\circ} \mathrm{C}$ to r.t., $12 \mathrm{~h}, 88 \%$; (f) 2,2-dimethoxypropane, PTS, r.t., 1 h, 90\%; (g) $\mathrm{O}_{3}, \mathrm{CH}_{2} \mathrm{Cl}_{2}$, $-78{ }^{\circ} \mathrm{C}, 2 \mathrm{~h}, 90 \%$; (h) $\mathrm{NaBH}_{4}, \mathrm{MeOH}, 0{ }^{\circ} \mathrm{C}, 20 \mathrm{~min}, 92 \%$; (i) $\mathrm{MsCl}$, $\mathrm{Et}_{3} \mathrm{~N}, \mathrm{CH}_{2} \mathrm{Cl}_{2}, 0{ }^{\circ} \mathrm{C}, 30 \mathrm{~min}, 85 \%$; (j) $\mathrm{NaN}_{3}$, DMF, r.t., 12 h, 81\%; (k) $\mathrm{PPh}_{3}, \mathrm{THF}, \mathrm{H}_{2} \mathrm{O}$, r.t., $2 \mathrm{~h}, 72 \%$; (I) $\mathrm{M} 4, t-\mathrm{BuCO}_{2} \mathrm{H}$, hexane, toluene, THF, $110{ }^{\circ} \mathrm{C}, 30 \mathrm{~h}$, then $2 \mathrm{~mol} / \mathrm{L} \mathrm{HCl}, 0{ }^{\circ} \mathrm{C}$ to r.t.; (m) $1 \mathrm{~mol} / \mathrm{L}$ $\mathrm{NaOH}$, THF, r.t., 6 h; then $1 \mathrm{~mol} / \mathrm{L} \mathrm{HCl}$ r.t., $67 \%$ (2 steps).

图式 2 阿托伐他汀的合成路线

Scheme 2 Synthetic route of atorvastatin

\section{2 结论}

我们从廉价易得的 $(R)$-2-氯甲基代环氧乙烷(5)为起 始原料, 经过 13 步反应, 以 $9 \%$ 的总收率成功地合成了 阿托伐他汀, 为相应的仿制药的生产提供了新的路线选
择. 需要指出的是, 在本合成路线中, 目标产物所需的 氮原子经由 $\mathrm{NaN}_{3}$ 参与的亲核取代反应及后续的 Staudinger 反应成功地引入，该策略成功地避免了大多 数 ATS-9 片段合成过程中 Raney-Ni 的使用, 不仅降低 了具体操作过程的危险性和繁琐度，同时也可以在后续 的中试放大过程中进一步降低对环境的影响 ${ }^{[18]}$.

\section{3 实验部分}

\section{1 仪器与试剂}

如无特殊说明, 实验所用溶剂使用前均按照标准方 法在氩气环境下进行处理, 重新蒸溜后使用; 所用药品 均从相应的试剂公司购买, 未经进一步纯化; ${ }^{1} \mathrm{H} \mathrm{NMR}$, ${ }^{13} \mathrm{C}$ NMR 均在 Bruker $\mathrm{AX}-400 \mathrm{MHz}$ 仪器中测定, $\mathrm{CDCl}_{3}$ 为溶剂, TMS 为内标; MS 采用岛津 2010 型质谱仪型仪 器测定, EI 源, $70 \mathrm{eV}$ 直接进样; 化合物旋光由相应的 $\mathrm{CDCl}_{3}$ 溶液测得 $(c=10 \mathrm{mg} / \mathrm{mL}) ;$; 薄层层析(TLC)和柱层 析分别使用山东青岛海洋化工厂生产的薄层层析硅胶 GF254 型硅胶和 200 300 目柱层析硅胶.

\section{2 实验方法}

\subsection{1 (2R)-1-氯-4-戊烯-2-醇(6)的合成}

在 $-20{ }^{\circ} \mathrm{C}$ 条件下, 于 $500 \mathrm{~mL}$ 圆底烧瓶中依次加入 干燥的四氢呋喃 $(200 \mathrm{~mL}), 1 \mathrm{~mol} / \mathrm{L}$ 乙烯基溴化镁的四氢 呋喃溶液 $(150 \mathrm{~mL}, 0.15 \mathrm{~mol})$ 和氯化亚铜 $(913 \mathrm{mg}, 0.01$ $\mathrm{mol})$. 保持温度不变, 搅拌 $20 \mathrm{~min}$ 后, 向体系中滴加 $(R)$-2-氯甲基代环氧乙烷 $(8 \mathrm{~mL}, 0.1 \mathrm{~mol})$. TLC 监测反应 完成后, 将反应体系用饱和的氯化铵溶液淬灭, 混合物 用乙酸乙酯萃取分离, 有机相经饱和食盐水洗涤后, 用 无水 $\mathrm{MgSO}_{4}$ 干燥、过滤. 滤液减压浓缩后经硅胶柱层析 分离(乙酸乙酯/石油醚作洗脱剂, 下同)得黄色油状液体 $6(11.6 \mathrm{~g}$, 产率 $91 \%) .[\alpha]_{\mathrm{D}}^{18}-3\left(c=1.0, \mathrm{CHCl}_{3}\right) ;{ }^{1} \mathrm{H} \mathrm{NMR}$ $\left(400 \mathrm{MHz}, \mathrm{CDCl}_{3}\right) \delta: 5.88 \sim 5.78(\mathrm{~m}, 1 \mathrm{H}), 5.21 \sim 5.16(\mathrm{~m}$, $2 \mathrm{H}), 3.95 \sim 3.85(\mathrm{~m}, 1 \mathrm{H}), 3.65(\mathrm{q}, J=3.6 \mathrm{~Hz}, 1 \mathrm{H}), 3.53$ $(\mathrm{dd}, J=11,2.6 \mathrm{~Hz}, 1 \mathrm{H}), 2.39 \sim 2.33(\mathrm{~m}, 2 \mathrm{H}) ;{ }^{13} \mathrm{C} \mathrm{NMR}$ $\left(100 \mathrm{MHz}, \mathrm{CDCl}_{3}\right) \delta: 133.3,118.7,70.6,49.4,38.7$; MS (EI) $m / z(\%): 120\left(\mathrm{M}^{+}, 1\right), 102(100), 85(20)$.

\section{$3.2 .2(2 R)-1$-氰基-4-戊烯-2-醇(7)的合成}

在 $200 \mathrm{~mL}$ 圆底烧瓶中分别加入 $100 \mathrm{~mL}$ 蒸馏水, 化 合物 6 (11.6 g, $0.92 \mathrm{~mol})$ 及氰化钠 $(6.8 \mathrm{~g}, 0.138 \mathrm{~mol})$. 反 应体系在 $30{ }^{\circ} \mathrm{C}$ 条件下搅拌过夜, 经 TLC 监测原料彻底 消失后，体系用乙酸乙酯 $(100 \mathrm{~mL})$ 萃取，分离后，有机 相用饱和食盐水洗涤, 无水 $\mathrm{MgSO}_{4}$ 干燥后过滤, 滤液减 压浓缩后经硅胶柱层析分离( 乙酸乙酯/石油醚作洗脱 剂) 得黄色油状液体 7 (8.4 g, 产率 $93 \%)$. $[\alpha]_{\mathrm{D}}^{18}+1(c=$ 1.0, $\mathrm{CHCl}_{3}$ ); ${ }^{1} \mathrm{H}$ NMR (400 MHz, $\left.\mathrm{CDCl}_{3}\right) \delta: 5.78 \sim 5.67$ 
(m, $1 \mathrm{H}), 5.15 \sim 5.10(\mathrm{~m}, 2 \mathrm{H}), 3.96 \sim 3.92(\mathrm{~m}, 1 \mathrm{H}), 3.30(\mathrm{~d}$, $J=4.8 \mathrm{~Hz}, 1 \mathrm{H}), 2.53(\mathrm{dd}, J=16.8,4.8 \mathrm{~Hz}, 1 \mathrm{H}), 2.44(\mathrm{dd}$, $J=16.8,6.0 \mathrm{~Hz}, 1 \mathrm{H}), 2.30$ (t, $J=6.6 \mathrm{~Hz}, 2 \mathrm{H}) ;{ }^{13} \mathrm{C} \mathrm{NMR}$ $\left(100 \mathrm{MHz}, \mathrm{CDCl}_{3}\right) \delta: 132.6,119.0,117.7,66.4,40.5,24.9$; MS (EI) $m / z$ (\%): $111\left(\mathrm{M}^{+}, 10\right), 83$ (50), 54 (100).

\subsection{3 (3R)-3-差基-5-已烯酸甲酯(4)的合成}

于 $200 \mathrm{~mL}$ 的圆底烧瓶中加入 $7(8.4 \mathrm{~g}, 0.74 \mathrm{~mol})$ 的 甲醇溶液 $(80 \mathrm{~mL})$. 回流条件下, 向反应体系中分三次 (间隔 $3 \mathrm{~h}$ ) 加入三甲基氯硅烷 $(48 \mathrm{~mL}, 1.14 \mathrm{~mol}$ ). 反应体 系继续回流反应至原料彻底消失 (TLC 监测). 反应完 毕, 减压条件下于旋转蒸发仪上除去反应溶剂, 剩余物 经柱层析 (乙酸乙酯/石油醚作洗脱剂) 得黄色油状液 体 $4\left(8.2 \mathrm{~g}\right.$, 产率 76\%). $[\alpha]_{\mathrm{D}}^{18}-9\left(c=1.0, \mathrm{CHCl}_{3}\right) ;{ }^{1} \mathrm{H}$ NMR $\left(400 \mathrm{MHz}, \mathrm{CDCl}_{3}\right): \delta 5.83 \sim 5.73(\mathrm{~m}, 1 \mathrm{H}), 5.10 \sim$ $5.06(\mathrm{~m}, 2 \mathrm{H}), 4.08 \sim 4.02(\mathrm{~m}, 1 \mathrm{H}), 3.66(\mathrm{~s}, 3 \mathrm{H}), 3.01(\mathrm{~s}$, $1 \mathrm{H}), 2.44$ (dd, $J=16.8,6.0 \mathrm{~Hz}, 1 \mathrm{H}), 2.40$ (dd, $J=16.0,8.4$ $\mathrm{Hz}, 1 \mathrm{H}), 2.26 \sim 2.22(\mathrm{~m}, 2 \mathrm{H}) ;{ }^{13} \mathrm{C} \mathrm{NMR}\left(100 \mathrm{MHz}, \mathrm{CDCl}_{3}\right)$ $\delta: 173.0,133.8,118.0,67.2,51.6,40.9,40.4 ; \mathrm{MS}$ (EI) $\mathrm{m} / \mathrm{z}$ (\%): $144\left(\mathrm{M}^{+},<1\right), 126(10), 103(40), 85(100)$.

\subsection{4 (5R)-3-羰基-5-着基-7-辛烯酸叔丁酯(3)的合成}

氩气条件下, 于 $250 \mathrm{~mL}$ 圆底烧瓶中依次加入干燥 的四氢呋喃 $(80 \mathrm{~mL})$, 二异丙胺 $(18.6 \mathrm{~mL}, 0.13 \mathrm{~mol})$. 在 $-78{ }^{\circ} \mathrm{C}$ 条件下, 向体系缓慢滴加 $2.5 \mathrm{~mol} / \mathrm{L}$ 的正丁基锂 (56.6 mL, $0.13 \mathrm{~mol})$, 搅拌 $10 \mathrm{~min}$ 后, 体系自然升温升至 $0{ }^{\circ} \mathrm{C}$ 继续反应 $30 \mathrm{~min}$. 之后将体系冷却至 $-78{ }^{\circ} \mathrm{C}$, 缓 慢向其中滴加乙酸叔丁酯 $(17.7 \mathrm{~mL}, 0.13 \mathrm{~mol})$ 的, 搅拌 $30 \mathrm{~min}$ 后, 加入化合物 $4(8.2 \mathrm{~g}, 0.056 \mathrm{~mol})$ 的四氢呋喃溶 液 $(20 \mathrm{~mL})$. 保持反应温度不变, 直至原料彻底消失 (TLC 监测), 然后, 将反应体系用氯化铵水溶液淬灭, 体系用乙酸乙酯萃取后分离. 有机相用饱和食盐水洗涤 后, 用无水 $\mathrm{MgSO}_{4}$ 干燥, 过滤, 滤液减压浓缩后经硅胶 柱层析分离(乙酸乙酯/石油醚作洗脱剂)得黄色油状液 体 $3\left(8.4 \mathrm{~g}\right.$, 产率 65\%). $[\alpha]_{\mathrm{D}}^{18}-3\left(c=1.0, \mathrm{CHCl}_{3}\right) ;{ }^{1} \mathrm{H}$ NMR $\left(400 \mathrm{MHz}, \mathrm{CDCl}_{3}\right) \delta: 5.86 \sim 5.76(\mathrm{~m}, 1 \mathrm{H}), 5.15 \sim$ $5.10(\mathrm{~m}, 2 \mathrm{H}), 4.18 \sim 4.12(\mathrm{~m}, 1 \mathrm{H}), 3.37(\mathrm{~m}, 1 \mathrm{H}), 2.80 \sim$ $2.61(\mathrm{~m}, 3 \mathrm{H}), 2.28 \sim 2.24(\mathrm{~m}, 2 \mathrm{H}), 1.48(\mathrm{~s}, 9 \mathrm{H}) ;{ }^{13} \mathrm{C} \mathrm{NMR}$ $\left(100 \mathrm{MHz}, \mathrm{CDCl}_{3}\right) \delta: 203.8,173.0,166.1,133.9,118.0$, 82.1, 67.2, 66.7, 51.6, 51.1, 48.7, 40.9, 40.4, 27.8; MS (EI) $m / z(\%): 228\left(\mathrm{M}^{+},<1\right), 71(40), 57(100)$.

3.2.5 (3R, 5R)-3,5-二羟基-7-辛烯酸叔丁酯 $(8)$ 的合成

氩气氛围下, 于 $200 \mathrm{~mL}$ 圆底烧瓶中将化合物 3 (8.4 $\mathrm{g}, 0.036 \mathrm{~mol})$ 加入到干燥的四氢呋喃 $(90 \mathrm{~mL})$ 与干燥的甲 醇 $(16 \mathrm{~mL})$ 的混合液中. 将体系冷却到 $-78{ }^{\circ} \mathrm{C}$ 后, 加入 二乙基甲氧基硼烷 $(5.76 \mathrm{~mL}, 0.05 \mathrm{~mol}$ ), 搅拌 $30 \mathrm{~min}$ 后, 向体系分批加入硼氢化钠 $(2.5 \mathrm{~g}, 0.066 \mathrm{~mol})$. 反应体系
在相同温度下, 摚拌至原料彻底转化后, 于旋转蒸发仪 上抽干溶剂后, 剩余物用乙酸乙酯 $(10 \mathrm{~mL})$ 溶解, 再加 入双氧水水溶液 $(15 \mathrm{~mL})$ 将嗍烷脱去, 混合体系用乙酸 乙酯 $(50 \mathrm{~mL})$ 萃取. 分离的有机相用饱和食盐水洗涤, 无 水 $\mathrm{MgSO}_{4}$ 干燥，过滤，滤液减压浓缩经硅胶柱层析分离 (乙酸乙酯/石油醚作洗脱剂)得黄色油状液体 8 (7.45 g, 产率 88\%). $[\alpha]_{\mathrm{D}}^{18}-15\left(c=1.0, \mathrm{CHCl}_{3}\right) ;{ }^{1} \mathrm{H}$ NMR $(400$ $\left.\mathrm{MHz}, \mathrm{CDCl}_{3}\right) \delta: 5.87 \sim 5.79(\mathrm{~m}, 1 \mathrm{H}), 5.15 \sim 5.11(\mathrm{~m}, 2 \mathrm{H})$, $4.26 \sim 4.21(\mathrm{~m}, 1 \mathrm{H}), 3.98 \sim 3.92(\mathrm{~m}, 1 \mathrm{H}), 2.42(\mathrm{~d}, J=6.4$ $\mathrm{Hz}, 2 \mathrm{H}), 2.28 \sim 2.45(\mathrm{~m}, 2 \mathrm{H}), 1.61 \sim 1.56(\mathrm{~m}, 2 \mathrm{H}), 1.45(\mathrm{~s}$, $9 \mathrm{H}) ;{ }^{13} \mathrm{C}$ NMR $\left(100 \mathrm{MHz}, \mathrm{CDCl}_{3}\right) \delta: 172.0,134.5,117.8$, 81.5, 71.2, 69.0, 42.6, 42.1, 41.6, 28.1; MS (EI) $\mathrm{m} / \mathrm{z}$ (\%): $230\left(\mathrm{M}^{+},<1\right), 115(30), 57$ (100).

3.2 .6 (3R,5R)-6-烯丙基-2,2-二甲基-1,3-二氧六环-4乙酸叔丁酯(9)的合成

室温下, 于 $50 \mathrm{~mL}$ 的圆底烧瓶中依次加入 2,2-二甲 氧基丙烷 $(10 \mathrm{~mL})$, 化合物 $8(7.4 \mathrm{~g}, 0.032 \mathrm{~mol})$ 和对甲苯 磺酸(270 mg, $0.0016 \mathrm{~mol})$, 混合物摚拌反应至 TLC 监测 原料彻底转化. 然后将体系于旋转蒸发仪上抽干溶剂后 柱层析得黄色油状液体 9 (7.82 g, 产率 $90 \%)$. [ $\alpha]_{\mathrm{D}}^{18}-10$ $\left(c=1.0, \mathrm{CHCl}_{3}\right) ;{ }^{1} \mathrm{H}$ NMR $\left(400 \mathrm{MHz}, \mathrm{CDCl}_{3}\right) \delta: 5.79 \sim$ $5.70(\mathrm{~m}, 1 \mathrm{H}), 5.06 \sim 5.00(\mathrm{~m}, 2 \mathrm{H}), 4.23 \sim 4.17(\mathrm{~m}, 1 \mathrm{H})$, $3.89 \sim 3.83(\mathrm{~m}, 1 \mathrm{H}), 2.38(\mathrm{dd}, J=15.2 \mathrm{~Hz}, 7.2 \mathrm{~Hz}, 1 \mathrm{H})$, $2.29 \sim 2.23(\mathrm{~m}, 2 \mathrm{H}), 2.14 \sim 2.07(\mathrm{~m}, 1 \mathrm{H}), 1.57 \sim 1.52(\mathrm{~m}$, 1H), 1.40 (brs, $12 \mathrm{H}), 1.32(\mathrm{~s}, 3 \mathrm{H}), 1.20 \sim 1.05(\mathrm{~m}, 1 \mathrm{H}) ;{ }^{13} \mathrm{C}$ NMR $\left(100 \mathrm{MHz}, \mathrm{CDCl}_{3}\right) \delta: 170.2,134.0,117.0,98.6$, 80.4, 68.4, 66.2, 42.7, 40.7, 35.9, 30.0, 28.0, 19.6; MS (EI) $m / z(\%): 255$ ([M-15] $\left.]^{+}, 10\right), 115$ (20), 57 (100).

$3.2 .7(3 R, 5 R)$-6-醛乙基-2,2-二甲基-1,3-二氧六环-4乙酸叔丁酯(2)的合成

在一78 ${ }^{\circ} \mathrm{C}$ 条件下, 向化合物 $9(7.82 \mathrm{~g}, 0.029 \mathrm{~mol})$ 的二氯甲烷 $(50 \mathrm{~mL})$ 溶液中持续通入臭氧, TLC 监测至 原料彻底转化后, 停止臭氧的通入, 向体系内加入三苯 基磷(11.73 g, $0.043 \mathrm{~mol}$ ), 室温下搅拌至反应中间体彻 底转化后, 于旋转蒸发仪上抽干溶剂后柱层析得黄色油 状液体 $2(7.1 \mathrm{~g}$, 产率 $90 \%) .[\alpha]_{\mathrm{D}}^{18}-28\left(c=1.0, \mathrm{CHCl}_{3}\right)$; ${ }^{1} \mathrm{H}$ NMR $\left(400 \mathrm{MHz}, \mathrm{CDCl}_{3}\right) \delta$ : $9.74(\mathrm{~s}, 1 \mathrm{H}), 4.24 \sim 4.28$ $(\mathrm{m}, 1 \mathrm{H}), 4.27 \sim 4.23(\mathrm{~m}, 1 \mathrm{H}), 2.61 \sim 2.54(\mathrm{~m}, 1 \mathrm{H}), 2.47 \sim$ $2.41(\mathrm{~m}, 2 \mathrm{H}), 2.27(\mathrm{dd}, J=15.2,6.0 \mathrm{~Hz}, 1 \mathrm{H}), 1.64 \sim 1.60$ (m, 2H), $1.44(\mathrm{~s}, 3 \mathrm{H}), 1.40(\mathrm{~s}, 9 \mathrm{H}), 1.35(\mathrm{~s}, 3 \mathrm{H}), 1.24 \sim$ $1.16(\mathrm{~m}, 1 \mathrm{H}) ;{ }^{13} \mathrm{C}$ NMR $\left(100 \mathrm{MHz}, \mathrm{CDCl}_{3}\right) \delta: 200.7$, 170.0, 99.1, 98.9, 80.6, 65.9, 64.5, 49.6, 42.4, 36.0, 29.8, 28.0, 19.5; MS (EI) $m / z$ (\%): $272\left(\mathrm{M}^{+},<1\right), 257$ (10), 157 (20), 57 (100). 
3.2.8 (3R, 5R)-6-羟基乙基-2,2-二甲基-1,3-二氧六环4-乙酸叔丁酯(10a) 的制备

$0{ }^{\circ} \mathrm{C}$ 下于 $50 \mathrm{~mL}$ 圆底烧瓶中依次加入甲醇 $(50 \mathrm{~mL})$ 、 化合物 $2(7.1 \mathrm{~g}, 0.26 \mathrm{~mol})$ 、嗍氢化钠 $(1.2 \mathrm{~g}, 0.03 \mathrm{~mol}, \mathrm{f}$ 分四批加入). TLC 监测原料彻底转化, 反应体系用水淬 灭, 乙酸乙酯 $(250 \mathrm{~mL})$ 萃取, 有机相用饱和食盐水洗涤, 无水 $\mathrm{MgSO}_{4}$ 干燥, 过滤, 浓缩后经柱层析后得 $\mathbf{1 0 a}(6.58$ $\mathrm{g}$, 产率 92\%). $[\alpha]_{\mathrm{D}}^{18}-15\left(c=1.0, \mathrm{CHCl}_{3}\right) ;{ }^{1} \mathrm{H}$ NMR $(400$ $\left.\mathrm{MHz}, \mathrm{CDCl}_{3}\right) \delta: 4.32 \sim 4.25(\mathrm{~m}, 1 \mathrm{H}), 4.18 \sim 4.11(\mathrm{~m}, 1 \mathrm{H})$, $3.82 \sim 3.73(\mathrm{~m}, 2 \mathrm{H}), 2.45(\mathrm{dd}, J=15.2,7.2 \mathrm{~Hz}, 1 \mathrm{H}), 2.32$ $(\mathrm{dd}, J=15.2,6.0 \mathrm{~Hz}, 1 \mathrm{H}), 1.76 \sim 1.72(\mathrm{~m}, 2 \mathrm{H}), 1.59 \sim 1.54$ (m, 1H), $1.48(\mathrm{~s}, 3 \mathrm{H}), 1.45(\mathrm{~s}, 9 \mathrm{H}), 1.38(\mathrm{~s}, 3 \mathrm{H}), 1.35 \sim$ $1.25(\mathrm{~m}, 1 \mathrm{H}) ;{ }^{13} \mathrm{C}$ NMR $\left(100 \mathrm{MHz}, \mathrm{CDCl}_{3}\right) \delta: 170.2,98.7$, 80.6, 68.4, 66.1, 60.1, 42.5, 38.2, 36.2, 30.0, 28.0, 19.7; MS (EI) $m / z$ (\%): 274 (M+ , <1), 273 (3), 257 (5), 217 (5), $173(5), 57(100)$.

3.2.9（3R,5R)-6-磺酸甲酯乙基-2,2-二甲基-1,3-二氧 六环-4-乙酸叔丁酯(10b)的制备

$0{ }^{\circ} \mathrm{C}$ 下于 $100 \mathrm{~mL}$ 的圆底烧瓶中依次加入二氯甲烷 $(30 \mathrm{~mL}) 、$ 化合物 $10 \mathrm{a}(6.58 \mathrm{~g}, 0.024 \mathrm{~mol})$ 和三乙胺 $(5.7$ $\mathrm{mL}, 0.042 \mathrm{~mol})$. 随后向上述体系中, 缓慢滴加甲烷磺酰 氯 $(3.2 \mathrm{~mL}, 0.042 \mathrm{~mol})$. 体系继续摚拌至原料彻底转化 后, 用水淬灭, 乙酸乙酯萃取, 有机相用饱和食盐水洗 涤, 无水 $\mathrm{MgSO}_{4}$ 干燥, 过滤, 滤液减压浓缩后经柱层析 得黄色油状液体 10b (7.18 g, 收率 85\%). $[\alpha]_{\mathrm{D}}^{18}+7$ ( $c=$ 1.0, $\mathrm{CHCl}_{3}$ ); ${ }^{1} \mathrm{H}$ NMR (400 MHz, $\left.\mathrm{CDCl}_{3}\right) \delta: 4.39 \sim 4.23$ (m, 3H), $4.06 \sim 4.00(\mathrm{~m}, 1 \mathrm{H}), 3.00(\mathrm{~s}, 3 \mathrm{H}), 2.43$ (dd, $J=$ $15.2,7.2 \mathrm{~Hz}, 1 \mathrm{H}), 2.30(\mathrm{dd}, J=15.2,6.0 \mathrm{~Hz}, 1 \mathrm{H}), 1.92 \sim$ $1.79(\mathrm{~m}, 2 \mathrm{H}), 1.61 \sim 1.56(\mathrm{~m}, 1 \mathrm{H}), 1.44(\mathrm{brs}, 12 \mathrm{H}), 1.35(\mathrm{~s}$, $3 \mathrm{H}), 1.25 \sim 1.17(\mathrm{~m}, 1 \mathrm{H}) ;{ }^{13} \mathrm{C} \mathrm{NMR}\left(100 \mathrm{MHz}, \mathrm{CDCl}_{3}\right) \delta$ : 170.1, 98.9, 80.7, 66.4, 66.0, 64.8, 42.6, 37.2, 36.3, 35.7, 30.0, 28.1, 19.7; MS (EI) $m / z(\%): 337\left([\mathrm{M}-15]^{+},<1\right)$, 337 (5), 257 (10), 221 (10), 115 (10), 57 (100).

3.2.10 (3R,5R)-6-叠氮乙基-2,2-二甲基-1,3-二氧六 环-4-乙酸叔丁酯(10c) 的制备.

室温下, 于 $25 \mathrm{~mL}$ 的圆底烧瓶中, 向化合物 $\mathbf{1 0 b}$ $(7.18 \mathrm{~g}, 0.02 \mathrm{~mol})$ 的 DMF $(10 \mathrm{~mL})$ 溶液中加入叠氮钠 $(2.66 \mathrm{~g}, 0.041 \mathrm{~mol})$. 反应体系摚拌至检测原料彻底转化 后, 用乙酸乙酯稀释, 有机相用水洗后, 无水 $\mathrm{MgSO}_{4}$ 干 燥, 过滤, 滤液减压浓缩后经柱层析得黄色油状液体 10c $(4.94 \mathrm{~g}$, 收率 $81 \%) .[\alpha]_{\mathrm{D}}^{18}+9\left(c=1.0, \mathrm{CHCl}_{3}\right) ;{ }^{1} \mathrm{H}$ NMR $\left(400 \mathrm{MHz}, \mathrm{CDCl}_{3}\right) \delta: 4.26 \sim 4.22(\mathrm{~m}, 1 \mathrm{H}), 4.00 \sim$ $3.95(\mathrm{~m}, 1 \mathrm{H}), 3.42 \sim 3.33(\mathrm{~m}, 2 \mathrm{H}), 2.42(\mathrm{dd}, J=15.2,6.8$ $\mathrm{Hz}, 1 \mathrm{H}), 2.29$ (dd, $J=15.2,6.4 \mathrm{~Hz}, 1 \mathrm{H}), 1.70 \sim 1.68(\mathrm{~m}$, $2 \mathrm{H}), 1.58 \sim 1.52(\mathrm{~m} .1 \mathrm{H}), 1.44(\mathrm{~s}, 3 \mathrm{H}), 1.43(\mathrm{~s}, 9 \mathrm{H}), 1.35(\mathrm{~s}$,
$3 \mathrm{H}), 1.24 \sim 1.18(\mathrm{~m}, 1 \mathrm{H}) ;{ }^{13} \mathrm{C}$ NMR $\left(100 \mathrm{MHz}, \mathrm{CDCl}_{3}\right) \delta$ : $170.1,98.8,80.6,66.1,65.8,47.5,42.6,36.4,35.6,30.0$, 28.1, 19.6; MS (EI) $m / z(\%): 257$ ([M-42] $\left.]^{+}, 5\right), 115$ (10), 57 (100).

3.2.11 (3R, 5R)-6-氨乙基-2, 2-二甲基-1, 3-二氧六环4-乙酸叔丁酯(ATS-9)的制备.

将化合物 10c (494 mg, $0.165 \mathrm{mmol}) 、 三$ 苯基膦(865 $\mathrm{mg}, 0.33 \mathrm{mmol})$ 加入到 $2 \mathrm{~mL}$ 的四氢呋喃与水 $(V: V=$ 9: 1)混合溶剂中, 摚拌至原料反应完全. 减压浓缩除 去溶剂, 向剩余物中加入 $2 \mathrm{~mL}$ 甲苯, 再次减压浓缩并 且重复俩次, 经柱层析得无色油状液体 ATS-9 (324 mg, 收率 $72 \%) .[\alpha]_{\mathrm{D}}^{18}+15\left(c=1.0, \mathrm{CHCl}_{3}\right) ;{ }^{1} \mathrm{H}$ NMR $(400$ $\left.\mathrm{MHz}, \mathrm{CDCl}_{3}\right) \delta: 4.30 \sim 4.20(\mathrm{~m}, 1 \mathrm{H}), 4.02 \sim 3.91(\mathrm{~m}, 1 \mathrm{H})$, $3.00 \sim 2.90$ (brs, $2 \mathrm{H}$ ), $2.90 \sim 2.80(\mathrm{~m}, 2 \mathrm{H}), 2.42$ (dd, $J=$ $15.2,7.2 \mathrm{~Hz}, 1 \mathrm{H}), 2.28(\mathrm{dd}, J=15.2,6.0 \mathrm{~Hz}, 1 \mathrm{H}), 1.66 \sim$ $1.52(\mathrm{~m}, 3 \mathrm{H}), 1.43$ (brs, $12 \mathrm{H}), 1.35$ (s, $3 \mathrm{H}), 1.21 \sim 1.15(\mathrm{~m}$, $1 \mathrm{H}) ;{ }^{13} \mathrm{C}$ NMR $\left(100 \mathrm{MHz}, \mathrm{CDCl}_{3}\right) \delta: 170.2,98.6,80.5$, 67.4, 66.2, 42.6, 39.2, 38.2, 36.5, 30.1, 28.0, 19.7; MS (EI) $m / z(\%): 273\left(\mathrm{M}^{+}, 1\right), 172(5), 57(100)$.

3.2.12 (3R,5R)-7-[2-(4-氟苯基)-5-异丙基-3-苯基4-(苯氨基甲酰基) 吡咯-1-基]-3,5-二羟基庚酸 (atorvastatin)的合成

将 ATS-9 (400 mg, $0.147 \mathrm{mmol}$ )、M4 (558 mg, 0.133 $\mathrm{mmol})$, 特戊酸 $(120 \mathrm{mg}, 0.118 \mathrm{mmol})$, 依次加入到正已 烷/甲苯/四氢呋喃 $(V: V: V=1: 4: 1,4.8 \mathrm{~mL})$ 的混合 溶剂中, 反应体系在 $110{ }^{\circ} \mathrm{C}$ 条件下加热 $30 \mathrm{~h}$ 后冷却至 室温, 混合物用乙酸乙酯稀释后加入饱和碳酸氢钠水溶 液萃取, 有机相用无水硫酸钠干燥后过滤, 滤液在旋转 蒸发仪上抽干溶剂. 将剩余物溶解在四氢呋喃 $(5 \mathrm{~mL})$ 中, 于 $0{ }^{\circ} \mathrm{C}$ 下加入 $2 \mathrm{~mol} / \mathrm{L}$ 的盐酸甲醇溶液 $(2 \mathrm{~mL})$, 在摚拌 $10 \mathrm{~min}$ 后升到室温搅拌 $20 \mathrm{~min}$, 将混合物用二氯甲烷稀 释后, 有机相用饱和碳酸氢钠与食盐水萃取, 无水硫酸 钠干燥、过滤, 滤液浓缩后用 $2 \mathrm{~mL}$ 四氢呋喃稀释, 在 $0{ }^{\circ} \mathrm{C}$ 下加入 $1 \mathrm{~mol} / \mathrm{L}$ 的氢氧化钠水溶液 $(10 \mathrm{~mL})$, 摚拌 10 $\min$ 后升到室温搅拌 $6 \mathrm{~h}$. TLC 监测原料反应完后, 用二 氯甲烷将混合物稀释, 加入 $1 \mathrm{~mol} / \mathrm{L}$ 盐酸水溶液至水相 显弱酸性, 分离有机相, 有机相用食盐水洗涤, 无水硫 酸钠干燥后过滤，滤液浓缩后经柱层析分离得无色油状 液体阿托伐他汀(548 mg, 产率 67\%). ${ }^{1} \mathrm{H}$ NMR (400 $\left.\mathrm{MHz}, \mathrm{CD}_{3} \mathrm{OD}\right) \delta: 7.30 \sim 7.01(\mathrm{~m}, 14 \mathrm{H}), 4.11 \sim 3.92(\mathrm{~m}$, $3 \mathrm{H}), 3.68 \sim 3.66(\mathrm{~m}, 1 \mathrm{H}), 3.40 \sim 3.36(\mathrm{~m}, 1 \mathrm{H}), 2.40 \sim 2.37$ $(\mathrm{m}, 2 \mathrm{H}), 1.72 \sim 1.60(\mathrm{~m}, 2 \mathrm{H}), 1.56 \sim 1.51(\mathrm{~m}, 1 \mathrm{H}), 1.49(\mathrm{~d}$, $J=7.2 \mathrm{~Hz}, 3 \mathrm{H}), 1.48(\mathrm{~d}, J=7.2 \mathrm{~Hz}, 3 \mathrm{H}), 1.47 \sim 1.40(\mathrm{~m}$, $1 \mathrm{H})$. 
辅助材料(Supporting Information) 化合物 2 4, 6

10 的 NMR 谱图. 这些材料可以免费从本刊网站 (http://sioc-journal.cn/)上下载.

\section{References}

[1] Global Status Report on Noncommunicable Diseases 2010, 2011, Geneva, World Health Organization (in Chinese). (2010 年全球非传染性疾病现状报告, 2011 年，日内瓦，世界卫 生组织.)

[2] Cai, Z.-Y.; Zhou, W.-C. Chin. J. New Drugs 2006, 15, 1907 (in Chinese).

(蔡正艳，周伟澄，中国新药杂志, 2006, 15, 1907.)

[3] Grouse, J. R.; Byington, R. P.; Hoen, H. M.; Furbzrg, C. D. Arch. Intern. Med. 1997, 157, 1305.

[4] Endo, A. J. Antibiot. 1979, 32, 852.

[5] Alberts, A. W.; Chen, J.; Kuron, G.; Alberts, A. W.; Chen, J.; Kuron, G.; Hunt, V.; Huff, J.; Hoffman, J.; Rothrock, J.; Lopez, M.; Joshua, H.; Harris, E.; Patchett, A.; Monaghan, R.; Currie, S.; Stapley, E.; AlbersSchonberg, G.; Hensens, O.; Hirshfield, J.; Hoogsteen, K.; Liesch, J.; Springer, J. Proc. Natl. Acad. Sci. 1980, 77, 3957.

[6] Roth, B. D. US 5273995, 1993 [Chem. Abstr. 1991, 115, 29107].

[7] Xu, S. Chin. New Drugs J. 2006, 15, 1913 (in Chinese).
(徐颂，中国新药杂志, 2006, 15, 1913.)

[8] Wang, J.-Y.; Shen, J.-F.; Wang, L.-X.; Wang, W.; Cai, Z.-G.; Du, Z.-J. Chin. J. Synth. Chem. 2007, 15, 519 (in Chinese). (王继宇, 沈健芬, 王立新, 王文, 蔡泽贵, 杜振军, 合成化学, 2007, 15, 519.)

[9] Wang, Z.-L.; Yin, J.; Lin, S.-C.; Yao, Z.-Y.; Deng, W.-P.; Du, W.-T. Chin. JMAP 2011, 28, 423 (in Chinese).

(王正林，应俊，林圣超，姚振宇，邓卫平，杜文婷，中国现代应 用药学杂志, 2011, 28, 423.)

[10] Brower, P. L.; Butler, D. E.; Deering, C. F.; Le, T. V.; Millar, A.; Nanninga, T. N.; Roth, B. D. Tetrahedron Lett. 1992, 33, 2279.

[11] Rádl, S.; Stach, J.; Hajicek, J. Tetrahedron Lett. 2002, 43, 2087.

[12] Gololobov, Y. G.; Zhmurova, I. N; Kasukhin, L. F. Tetrahedron 1981, 37, 437.

[13] Schuda, A. D. C.; Mazzocchi, P. H.; Fritz, G.; Morgan, T. Synthesis 1986, 309.

[14] Basu, M. K.; Luo, F.-T. Tetrahedron Lett. 1998, 39, 3005.

[15] Butler, D. E.; Le, T. V.; Millar, A.; Nanninga, T. N. US 5155251, 1992 [Chem. Abstr. 1993, 118, 41188].

[16] Tatsuya, M.; Kyoko, T.; Tamejiro, H. Tetrahedron Lett. 1993, 34, 513.

[17] Kawato, Y.; Iwata, M.; Yazaki, R.; Kumagai, N.; Shibasaki, M. Tetrahedron 2011, 67, 6539.

[18] 专利申请审核中(中国专利申请, 编号 201410491818.8).

(Zhao, X.) 